\title{
Role of CT Angiography in Comparison to Ultrasound for Detection, Characterization and Quantification of Carotid Artery Disease
}

\author{
Mayankkumar Patel ${ }^{1}$, Jaival Dave ${ }^{2}$, Asutosh Dave ${ }^{3}$, Jaideep Jagani ${ }^{4}$ \\ ${ }^{1} 2^{\text {nd }}$ Year Resident, Department of Radiodiagnosis, GCS Medical College, ${ }^{2}$ MBBS, Smt. N.H.L Municipal Medical College, \\ ${ }^{3}$ Professor and Head, Department of Radiodiagnosis, GCS Medical College, ${ }^{4} 2^{\text {nd }}$ Year Resident, Department of Radiodiagnosis, \\ GCS Medical College, India
}

Corresponding author: Dr.Mayankkumar Patel, 7, Sambhavnath Society, Gayatri Mandir Road, Mahavirnagar, Himmatnagar, Dist-Sabarkantha 383001, India

DOI: http://dx.doi.org/10.21276/ijcmsr.2019.4.2.41

How to cite this article: Mayankkumar Patel, Jaival Dave, Asutosh Dave, Jaideep Jagani. Role of CT angiography in comparison to ultrasound for detection, characterization and quantification of carotid artery disease. International Journal of Contemporary Medicine Surgery and Radiology. 2019;4(2):B185-B187.

\section{A B S T R A C T}

Introduction: Stroke is the third most common cause of death in developed word. About $80 \%$ strokes are ischemic in nature. Extracranial carotid artery atherosclerosis is third leading cause of ischemic stroke in general population. CT Angiography (MDCTA) and Ultrasound color doppler (USCD) are two frequently employed modalities for evaluation of carotid artery disease. The purpose of this study was to assess effectiveness of CTA compare to USCD in detection, characterization and quantification of carotid artery disease.

Material and Methods: Study was conducted in GCS Medical college on fifty patients. MDCTA was performed in patients in which carotid Doppler ultrasound was suggestive of $>50 \%$ stenosis and high-risk plaque such as ulcerated plaque, irregular surface plaque.

Results: Our study shows that CTA angiography is better modality compare to USCD in quantification of stenosis and plaque characterization.

Conclusion: USCD should be first line investigation for carotid artery disease. However due to less sensitivity of USCD in determining stenosis and some plaque characteristics CTA should be performed for detailed plaque evaluation.

Keywords: Stroke, Doppler Ultrasound, CT Angiography, Carotid Artery Disease

\section{INTRODUCTION}

Stroke is the third most common cause of death in developed word. About $80 \%$ strokes are ischemic in nature. Extracranial carotid artery atherosclerosis is third leading cause of ischemic stroke in general population. ${ }^{1}$

CT Angiography (MDCTA) and Ultrasound color doppler (USCD) are two frequently employed modalities for evaluation of carotid artery disease. MDCTA is generally used after USCD has analyzed underlying pathology. USCD is cost effective screening examination of carotid artery without risk of ionizing radiation and contrast related adverse reaction. ${ }^{2}$

Quantification of carotid artery stenosis is required for surgical management of carotid artery disease. Three studies- NASCET, ECST and ACAS has demonstrated effect of endarterectomy in symptomatic patient and provided cut off value for carotid artery stenosis in which surgical management required. ${ }^{3}$

Recent work on carotid artery disease suggests need of evaluation of plaque morphology for better treatment planning. Plaque morphology like ulceration (ulcerated, nonulcerated), type of plaque (fatty, calcified, mixed) are important parameter to determine risk of plaque rupture and subsequent thromboembolism. ${ }^{4}$ The purpose of this study was to assess effectiveness of CTA compare to USCD in detection, characterization and quantification of carotid artery disease.

\section{MATERIAL AND METHODS}

Study was done in Department of Radiology in GCS Medical college. Fifty patients (100 carotid arteries) were included in study during the period of February 2018 to July 2018. All patients were referred from tertiary care hospital for Carotid Doppler ultrasound for suspected atherosclerotic disease. Detailed medical history regarding risk factors (Diabetes, hypertension, altered lipid profile, smoking, obesity) and current symptoms (no symptoms, TIA, Paralysis) were taken. MDCTA was performed in patients in which carotid doppler ultrasound was suggestive of $>50 \%$ stenosis and high-risk plaque such as ulcerated plaque, irregular surface plaque.

MDCTA was also performed in those patients in whom examination by USCD was limited by obesity, short neck, higher bifurcation of carotid artery and calcified plaques. Exclusion criteria for the study were allergy to contrast 
media or elevated serum creatinine level.

\section{US Technique}

Bilateral carotid Doppler ultrasound was performed in all patients by general electric ultrasound machine (GE LOGIQ P5) using linear $10 \mathrm{MHz}$ transducer. Patients were made to lie down in supine or semi supine position with head hyperextended and rotated $45^{*}$ away from side being examined.

Doppler examination includes gray scale examination, color Doppler, power Doppler and spectral Doppler examination of CCA, ICA, ECA and vertebral artery. Site for Doppler traces in CCA were proximal and distal part. Site for Doppler traces in ICA were proximal, middle and distal part. Site for Doppler traces in ECA was proximal part. Site for Doppler traces for vertebral artery was V0-V1-V2. Area of Carotid bulb and maximum stenosis were also included for sampling. Color gain, the angle of insonation, wall filtered, PRF were adjusted according the need. Maximum blood velocity, peak systolic velocity (PSV), end diastolic velocity (EDV) was measured in all patients. When stenosis was present maximum velocity through that area also recorded. The lesions were evaluated for echogenicity, calcification and plaque surface features.

\section{CTA Technique}

All patients went for angiography of carotid vessels using Siemens 16 slice SOMATOM Siemens system. Written and informed consent was taken regarding contrast related side effect and its potential benefits. Patient was placed in supine position with head tilted back. Patients were asked to hold breath. Contrast was injected through cubital vein. Region of interest (ROI) and FOV were set accordingly. Obtained data was processed with workstation to create MPR, MIP and volume rendered post processed images.

\section{Measurement method}

A single radiologist blinded to clinical information performed all measurement. The stenosis of carotid artery was graded using NASCET trial criteria by measuring residual luminal surface at the stenosis and surface of the distal normal lumen where there is no stenosis.

\section{RESULTS}

Out of 100 carotid arteries (CA) of 50 patients, $37 \mathrm{CA}$ shows no stenosis on CDUS, 3 CA shows $1-49 \%$ stenosis of ICA, 26 CA shows 50-69\% stenosis, 15 CA shows 70-79\% stenosis, 19 CA shows $100 \%$ stenosis by CDUS (Image-1). 63 CA shows plaque ulceration. Out of 63 CA, 59 CA shows no ulcer and 4 CA shows ulceration on CDUS. On CTA 57 CA shows no ulcer while 6 CA shows ulcer. Out of $63 \mathrm{CA}$, $37 \mathrm{CA}$ shows soft plaque,16 CA shows mixed plaque, $10 \mathrm{CA}$ shows calcified plaque on CDUS (Image $2 \& 3$ ). On CTA 32 CA shows soft plaque, $24 \mathrm{CA}$ shows mixed plaque and $7 \mathrm{CA}$ shows calcified plaque. Out of 100 patient $37 \mathrm{CA}$ shows no stenosis on CTA, 5 CA show 1-49\% stenosis of ICA, 22 CA show $50-69 \%$ stenosis, 18 CA show 70-79\% stenosis, 18 CA show $100 \%$ stenosis by CTA (Image- 4 ).

Study findings suggest CDUS is better modality to detect and quantify carotid artery plaque characterization and ulceration while CTA is better modality to detect carotid artery

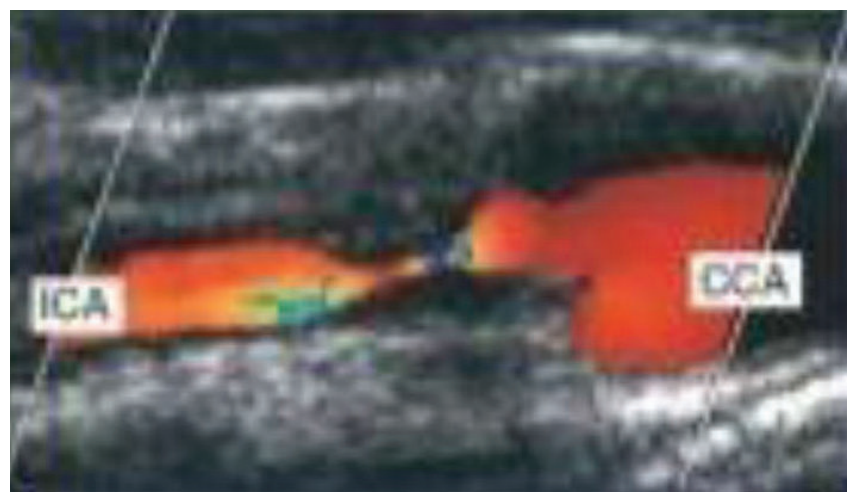

Image-1: ICA stenosis: PSV: $500 \mathrm{~cm} / \mathrm{s}, \mathrm{EDV}: 300 \mathrm{~cm} / \mathrm{s}$, Spectral broadening, 80\% Diameter stenosis

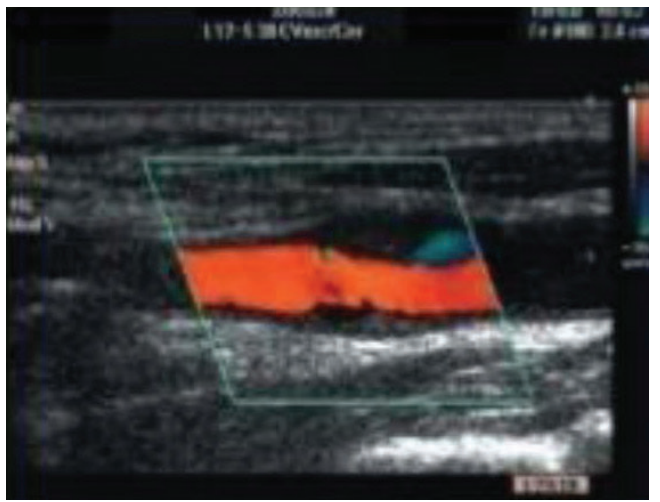

Image-2: Plaque characteristics - Homogeneous echo lucent

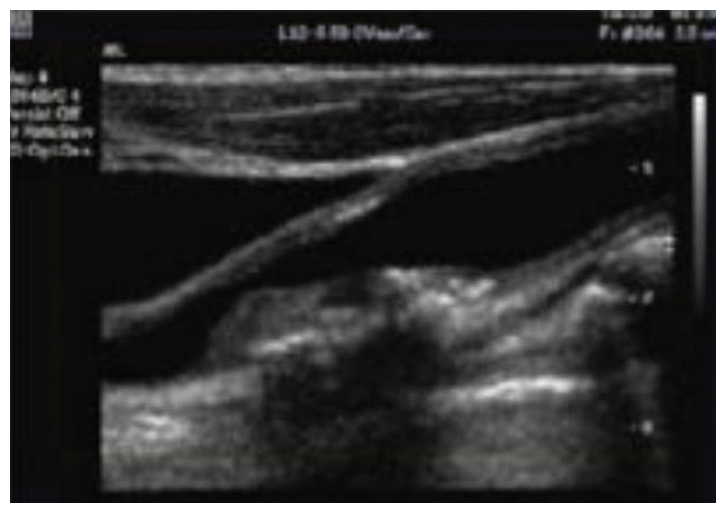

Image-3: Heterogeneous echogenic

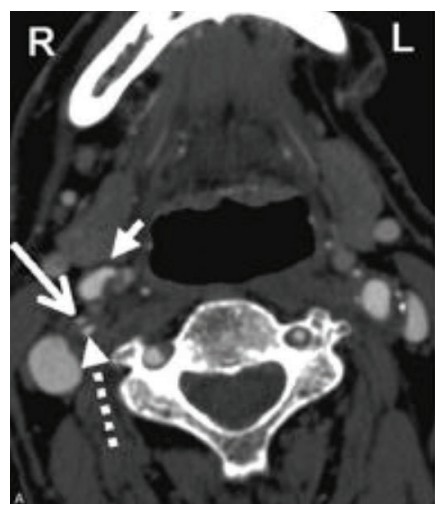

Image-4: CTA showing right severe carotid bulb stenosis with deep plaque ulceration. (Large arrow, stenotic ICA; dashed arrow, bulb plaque ulcer; small arrow, proximal ECA). 
stenosis. Study conducted by Deberenardi $\mathrm{S}$ et $\mathrm{al}^{9}$ suggest CTA is better modality to detect plaque ulceration while our study suggest CDUS is better modality to characterize plaque ulceration. Study also suggested CTA is superior for detection of stenosis, the finding which was consistent with our study.

\section{DISCUSSION}

Stroke is the commonest cause of mortality after coronary artery disease worldwide. It is also commonest cause of adult disability. The stroke has significant economic burden on society. The disability adjust life year (DALY) is currently the most important time-based measure of chronic disease including both disability and mortality. Accordingly, global data from 2004 covering 192 WHO members countries, stroke related DALY loss in India was 596.6 per 100000 persons per year. $^{5}$

The result of two randomized trials-NASCET \& amp; ECT have shown carotid endarterectomy give considerable benefits in patient with 70-99\% stenosis and small benefits in $50-69 \%$ stenosis. ${ }^{6}$ CTA is gold standard technique used to quantify the carotid artery stenosis. But it has some limitation like increase risk of thromboembolic events, contrast related nephropathy and risk of radiation. ${ }^{7}$

MRA has shown promising results but has limitation like overestimation of stenosis, less sensitivity in detection of ulceration, less coverage of anatomic area, flow dependence especially when performed without contrast. ${ }^{8,9}$

USCD is safe and easy to perform compared to CTA and widely used to detect carotid artery stenosis and plaque characterization but has less sensitivity and specificity for detection of carotid artery stenosis. Presence of heavy calcification and higher bifurcation of carotid artery may limit its use.

Our study shows CTA angiography is better modality compare to USCD in quantification of stenosis, plaque characterization.

\section{CONCLUSION}

USCD should be first line investigation for carotid artery disease. However due to less sensitivity of USCD in determining stenosis and some plaque characteristics CTA should be performed for detailed plaque evaluation.

\section{REFERENCES}

1. Ooi, Y. C., Gonzalez, N. R. Management of extracranial carotid artery disease. Cardiology clinics 2015;33:1-35.

2. Steinke W, Kloetzsch C, Hennerici M. Carotid artery disease assessed by color Doppler flow imaging: Correlation with standard Doppler sonography and angiography. AJR Am J Roentgenol. 1990;154:1061-8

3. Saba L, Mallarini G. A comparison between NASCET and ECST methods in the study of carotids: evaluation using Multi-Detector-Row CT angiography. Eur J Radiol. 2010;76:42-7.

4. Sun, R., Wang, L., Guan, C., Cao, W. and Tian, B. Carotid Atherosclerotic Plaque Features in Patients with Acute Ischemic Stroke. World Neurosurg. 2018;112:e223-e228.

5. Banerjee, T. K., Das, S. K. Fifty years of stroke researches in India. Annals of Indian Academy of Neurology 2016;19:1-8.

6. Kılıçkap G, Ergun E, Başbay E, Koşar P, Kosar U. Carotid stenosis evaluation by 64-slice CTA: comparison of NASCET, ECST and CC grading methods. Int J Cardiovasc Imaging. 2012;28:1257-66

7. Tavakol, M., Ashraf, S., Brener, S. J. Risks and complications of coronary angiography: a comprehensive review. Global journal of health science 2012;4:65-93.

8. Patel MR, Klufas RA, Kim D, Edelman RR, Kent KC. MR angiography of the carotid bifurcation: artifacts and limitations. AJR Am J Roentgenol. 1994;162:1431-7.

9. Debernardi S, Martincich L, Lazzaro D, Comelli S, Raso AM, Regge D. CT angiography in the assessment of carotid atherosclerotic disease: results of more than two years' experience. Radiol Med. 2004;108:116-27.

Source of Support: Nil; Conflict of Interest: None

Submitted: 10-04-2019; Accepted: 10-05-2019; Published online: 30-06-2019 